\title{
Limits of spectral resolution in optical measurements
}

\author{
Manuel B. Marques* \\ INESC Porto and Department of Physics and Astronomy of the University of Porto, Rua do Campo \\ Alegre, 687, 4169-007 Porto, Portugal
}

\begin{abstract}
Nowadays a growing number of scientists relies on optical spectral measurements for their research. The market is full of new plug-and-play equipment for spectral analysis that take the fuss out of the measurements. As with other instruments (computers, lasers, etc.) the researcher doesn't need any longer to work with someone with a post-graduate formation on the technology to be able to do excellent research. But, as in every instrument, there are limitations on the instrument use that affect its precision and resolution.

Currently there is in the market a large variety of equipment for spectral measurements. They range from the huge long focal length double pass monochromators to the small pocket size USB connected array spectrometers. The different configurations have different sensitivities on the light input system, light intensity, coherence, polarization, etc.
\end{abstract}

In this talk we will discuss a few of the limitations in spectral measurements that can be found in experimental setups.

Keywords: Resolution, Spectroscopy

\section{INTRODUCTION}

Light spectra have always amazed mankind. Rainbows and halos took a special treatment in mythology and religion. But only in the last two centuries became important in science. The first historical report of spectroscopy was the work of Wollaston published in $1802^{1}$ where he reported the dark lines in the solar spectrum. He based his work on the observations made by Newton (1666) and Bacon (13th century) in the dispersion of the solar light by glass and water prisms.

Fraunhofer (for many, considered the father of spectroscopy) brought spectroscopy to the frontline of science with its invention of the spectroscope in 1814 and of the diffraction grating in 1821.

The developments in the second half of the 19th century were amazing: identification of new elements, chemical analysis, and the beginning of atomic theory. The evolution of spectroscopy continued during the 20th century, with new developments, bringing spectroscopy to the 21 th century as one of the most influential tool in research.

Due to the widespread use of spectroscopy in a broad range of sciences, most researchers are unaware of its limitations or its basic operational principles. A basic review of spectroscopy principles is a must prior to work in spectroscopy, fortunately there are good reviews directed for each area ${ }^{2,3}$.

In this communication we intend to present some of the limitations in resolution posed by spectrometer construction. We will consider only spectroscopes build with a grating, although its transposition to prism spectroscopes can be straight forward, and exclude other types of spectral measurement instruments, like Fabry-Perot interferometers. For simplicity we will limit the discussion to the Littrow configuration. To consider other configurations, the angle $\theta$ in the following equations should be replaced by the mean of the incident and the refracted angles $\left(\theta=\left(\theta_{i}+\theta_{r}\right) / 2\right)$.

\subsection{Spectral resolution}

For a grating spectroscope, or monochromator, in the Littrow configuration, the exit wavelength obeys the equation ${ }^{4}$

$$
2 a \sin \theta=m \lambda
$$

where $a$ is the grating groove separation, $\theta$ is the incident angle in the grating, $m$ is the diffraction order and $\lambda$ is the wavelength of light.

*mbmarque@fc.up.pt 
The resolving power $(R)$ of a spectrometer in this configuration, as can be seen in any elementary optics textbook ${ }^{4}$, is given by:

$$
R=\frac{\lambda}{\Delta \lambda_{\min }}=\frac{2 N a \sin \theta}{\lambda}=m N
$$

where $\Delta \lambda_{\min }$ is the minimum separation between two spectral lines in the Rayleigh limit and $N$ is the total number of grooves in the grating.

This equation shows that the theoretical resolution depends exclusively on the number of grooves illuminated and on the order of diffraction. The equation is deduced considering the spot size of the light focused at the output, obtained in the diffraction limit, and the linear dispersion that depends on the groove density, on the diffraction order and on the focal length. This equation is valid even for double or triple monochromators. However, in most situations, this limit is not achieved. In the following points we will enumerate several limitations that don't allow for reaching this resolution.

\section{Size}

The linear dispersion is proportional to the focal length of the system, so usually longer monochromators have higher resolution, for the same spot size. The diffraction limit of the spot size depends on the focal distance $(f)$ and on the beam diameter (D) and is commonly referred through the f-number $(f / \#=f / D)$ of the focusing system. The diameter of the Airy disc is roughly $2.44 \lambda f / D$ and the corresponding Rayleigh distance is in the order of $4.7 \lambda(f / D)^{2}$. For a typical system with an f-number of 2, working at $500 \mathrm{~nm}$, we will get a spot diameter of $2.4 \mu \mathrm{m}$ and a Rayleigh distance of 9.4 $\mu \mathrm{m}$. Although the optical power decreases with the square of the f-number, its value is usually in the range 2-10 due to the aberrations and tolerance limitations in the construction. As a conclusion, size matters!

\section{Entrance and output slits}

Every spectrometer, monochromator or optical spectrum analyzer (OSA), have an entrance slit and an output slit (or the pixel size). We have assumed that the entrance and exit slits are small enough to be disregarded. If the slits have to be taken into account, a convolution between the spot size, due to the imaging system, and the slits sizes must be performed prior to the analysis. Usually most spectroscopes have a slit size smaller than the spot size to avoid this problem, but when using a different input condition (for instance using a multimode fiber at the entrance of an OSA) resolution will decrease.

Focusing light to a spot smaller than the input slit with very low numerical aperture $(N A \approx 1 /(2 f / \#)$ leads to a lower resolution since the grating is not filled with light, equivalent to a smaller grating. So never use a visible monomode fiber (typical values: field diameter $\sim 4 \mu \mathrm{m}$, N.A. $~ 0.12$ ) to input light to a large monochromator with small f-number and tens of microns entrance slit.

\section{Thermal fluctuations}

For very high resolution spectroscopy the temperature control of the monochromator is a must. Thermal variations of the system will affect the output wavelength in two ways. First, if there is any change in the position of the output slit, or the rotation angle of the grating, due to thermal expansion of the mechanical components, there will be an obvious error in the wavelength. This error can be estimated, and compensated, by the use of a spectral reference. But a second effect is more difficult to overpass, that is the change in the grating spacing. For this case only a two wavelength calibration can be effective. The error expected is in the order of the thermal expansion coefficient of the grating material. For glass the values range from (4 to 10$) \times 10^{-6} \mathrm{~K}^{-1}$, meaning that an increase of $10 \mathrm{~K}$ gives an error in the order of 0.5 angstrom at 500 nm wavelength.

\subsection{Power resolution}

Measuring high dynamical ranges in spectra have also some design limitations that must be taken into consideration to get reliable results. Usually most spectrometers are reliable in a range of three to six optical densities (OD). To go over this one in a million limit special care must be taken with stray light, second order diffraction rejection and detector linearity. Even the instrument design can lead to wrong results when high dynamical range is achieved in a short wavelength range 5 .

\section{Stray light}

One of the main sources of error in spectrometry is the stray light inside the instrument. In theory only light from a small range of wavelengths will exit through the exit slit (or impinges on one pixel of the CCD array). But not all the other light entering the input slit is absorbed by the blacked walls of the instrument; there is always a small fraction of 
unwanted light reaching the output. Of course it is easier in the monochromator design to have a smaller amount of stray light than in CCD based instruments.

The reduction of stray light inside the instrument, made either by placing internal shields and/or by the use of special paints, is one of the most important aspects in the design of high quality spectrometers. For low yield fluorescence measurements, where high energy pump light is important (like Raman spectroscopy), extra care must be taken with the stray light. In these measurements, as the pump light is nearly monochromatic, usually a matched band blocking filter is used at the spectrometer input.

Another source of stray light is due to small imperfections in the grating manufacturing, which creates small side lobes in the transfer function.

\section{Second order diffraction}

Equation 1 shows one of the big issues in grating monochomators. The second order diffraction of light at wavelength $\lambda_{1}$ will superimpose in space with the light from wavelength $\lambda_{2}=2 \lambda_{1}$. This can be avoided using prism spectroscopy, with nonlinear resolution and weight problems, but is usually overcome by the use or bandpass filters. For cheap CCD spectrometers, the blocking filters can be deposited over the CCD itself. The solution based on bandpass filters transfers the problem to the filter industry. High OD edge filters are difficult to fabricate and expensive, its manipulation is also delicate since any stress in the mounting ring could affect its spectral response. As an example, a $10 \mu \mathrm{m}$ pinhole in the multilayer filter, with a $1 \mathrm{~cm}$ beam, will reduce its blocking capacity to less than $6 \mathrm{OD}$.

Using high blocking instruments is a must when high dynamical range measures are needed and the input light is broad band.

\section{Detector nonlinearity}

For high intensities detectors behave nonlinearly as they approach saturation. On the other hand, very weak signals can be below the noise floor of the detector. To reduce the first problem it is usual to insert calibrated attenuators when measuring near the high power wavelengths, the drawback of this approach is that the weak features in the proximity of the strong one are also attenuated, a second problem is the wavelength calibration of the attenuator. The second aspect is more difficult to cope with; increasing the light throughput can be obtained by changing three parameters, increasing light power at the input, increasing f-number, reducing resolution by opening the slits. Usually none of these is a feasible option in a limiting experiment.

\subsection{Other limitations}

\section{Very short pulses}

For very short pulses we have to reformulate all formulae for spectral resolution. As an example consider a $10 \mathrm{fs}$ pulse at $600 \mathrm{~nm}$ in a spectrometer in the Littrow configuration. The grating, in the first order, will interfere light that has a temporal overlapping; after calculation, only the light diffracted from five grooves will be superimposed in time. For this example the diffraction pattern of the diffraction grating must be replaced by the diffraction pattern of a five slit system with its five maxima in the free spectral range.

This will lead to an increase of the width of the principal maxima and an increase of the stray light (the second maxima is only $1 / 22$ smaller than the principal one). As the pulse length increases, more groves are interfering and resolution increases. As an example for a $12 \mathrm{ps}$ pulse, at $600 \mathrm{~nm}$, resolution will be around 1 angstrom.

\section{Cosmic spikes}

Sometimes a huge value is detected in a single measurement at a particular wavelength value. Repeating the measurement shows that it was a noise feature. These huge values are known as cosmic spikes since in some types of detectors they can be produced through a high energetic cosmic ray. For sensitive measurements, even with high averaging, this feature can give a value order of magnitude higher than the real value.

The only way to avoid this effect is a manual (or automatic) removal of the affected points prior to the averaging.

\section{Self-fluorescence}

Some samples, even filters, have fluorescence. This will convert light from one wavelength to another one, leading to errors. This effect is usually important in absorbance measurements; here it is reduced by using a monochromator setup, where the sample is measured with monochromatic light. Self-fluorescence of parts inside the spectrometer is also a source of stray light. 


\section{CONCLUSIONS}

Spectral measurements became very important in several scientific domains and researchers are often unaware of the basic principles of operation. Most users of spectral instruments do not have formation in Optics and believe that the spectrum output is a rigorous representation of the real spectrum even when pushing the instrument to its limits.

At normal use, the instrument specifications hold, and we can use it at ease, but in many experiments we use the instrument at a different input configuration. Spectra measurements at the instrument limits can lead to errors if the operator is not aware of these limitations.

In this talk we presented the limits that we consider the most important to a researcher be aware of when making spectral measurements.

\section{REFERENCES}

[1] Wollaston, William Hyde, "A method of examining refractive and dispersive powers, by prismatic reflection," Philosophical Transactions of the Royal Society, 92, 365-380 (1802).

[2] Lerner, Jeremy M., "A method of examining refractive and dispersive powers, by prismatic reflection," Cytometry Part A, \&)A, $712-734$ (2006).

[3] Owen, Tony, [Fundamentals of modern UV-visible spectroscopy], Agilent Technologies, (2000)

[4] Hecht, Eugene, [Optics], $3^{\text {rd }}$ edition, Addison-Wesley, New York, 465-472 (1998).

[5] Prabhat, Prashant and Erdogan, Turan, "Measurement of Optical Filter Spectra," Semrock Technical Note, <http://www.semrock.com/measurement-of-optical-filter-spectra.aspx > (15 April 2014). 